\title{
Periprosthetic Knee Infection: Ten Strategies That Work
}

\author{
Javad Parvizi, MD, Priscilla Ku Cavanaugh, MS, and Claudio Diaz-Ledezma, MD \\ Department of Orthopaedic Surgery, Thomas Jefferson University Hospital, Philadelphia, PA, USA
}

Periprosthetic joint infection (PJI) is one of the most serious complications following total knee arthroplasty (TKA). The demand for TKA is rapidly increasing, resulting in a subsequent increase in infections involving knee prosthesis. Despite the existence of common management practices, the best approach for several aspects in the management of periprosthetic knee infection remains controversial. This review examines the current understanding in the management of the following aspects of PJI: preoperative risk stratification, preoperative antibiotics, preoperative skin preparation, outpatient diagnosis, assessing for infection in revision cases, improving culture utility, irrigation and debridement, one and twostage revision, and patient prognostic information. Moreover, ten strategies for the management of periprosthetic knee infection based on available literature, and experience of the authors were reviewed.

Keywords: Knee, Arthroplasty, Periprosthetic joint infection, Infection control, Reoperation

\section{Introduction}

It is well recognized that a periprosthetic joint infection (PJI) after knee arthroplasty is a catastrophic complication not only for the patient, but also for the health-care system. During the last decade, clinical research has considerably improved our comprehension of this topic. However, we are still in the process of producing high-level evidence to support our daily clinical practice. In this article we will review ten strategies that work in managing knee PJI.

\section{Preoperative Risk Stratification}

From our standpoint, the first step to succeed in the battle against knee PJI is prevention. Consequently, in order to stratify the patient's risk, knowledge of risk factors in the development

Received September 11, 2013; Revised November 6, 2013;

Accepted November 7, 2013

Correspondence to: Javad Parvizi, MD

Department of Orthopaedic Surgery, Thomas Jefferson University

Hospital, 111 S 11th St, Philadelphia, PA 19107, USA

Tel: +1-267-339-7813, Fax: +1-215-503-5651

E-mail: parvj@aol.com

This is an Open Access article distributed under the terms of the Creative Commons Attribution Non-Commercial License (http://creativecommons.org/licenses/by-nc/3.0/) which permits unrestricted non-commercial use, distribution, and reproduction in any medium, provided the original work is properly cited. of PJI should be mandatory for each surgeon that is involved in knee arthroplasty. Optimization of modifiable variables that may influence this risk is also critical. A recent study by Namba et al. ${ }^{1)}$ evaluated 56,216 total knee arthroplasty (TKA) surgeries. The study found an incidence of deep infection of $0.72 \%(404 / 56,216)$. The risk factors for infection were body mass index (BMI) of $\geq 35$ (hazard ratio [HR], 1.47), diabetes mellitus (HR, 1.28), male sex (HR, 1.89), an American Society of Anesthesiologists (ASA) score of $\geq 3$ (HR, 1.65), osteonecrosis ( $\mathrm{HR}, 3.65$ ), and posttraumatic arthritis (HR, 3.23). Hispanic race was a protective factor (HR, 0.69), the use of antibiotic irrigation ( $\mathrm{HR}, 0.67)$, a bilateral procedure (HR, 0.51), and a lower annual hospital volume (HR, 0.33). Surgical risk factors included quadriceps-release exposure (HR, 4.76), and the use of antibiotic-laden cement (HR, 1.53). Operative time was a risk factor, with a $9 \%$ increased risk per fifteen-minute increments. Although some risk factors found in this study are debatable, the study highlights some of the important predisposing factors for infection.

Obesity and diabetes are both well-known risk factors for knee PJI. According to the American Association of Hip and Knee Surgeons (AAHKS), prior to surgery, a patient with a BMI over 40 should be counseled regarding weight loss $^{2}$. In addition, AAHKS has emphasized the importance of a nutritional evaluation prior to surgery ${ }^{2}$. Regarding diabetes, a large Finnish study corroborated its relevance as a strong risk factor for infections ${ }^{3)}$. Although Hemoglobin A1C has been used to evaluate arthro- 
plasty patients, a recent publication from Iorio et al. ${ }^{4)}$ showed that it is probably a suboptimal preoperative measurement due to its inability to prognosticate complications. We believe that an exhaustive preoperative evaluation, and glycemic level optimization is an indispensable component of the preoperative work up, and must be conducted by a general internist, or medicine subspecialist. Currently, the evidence is not able to present a definite threshold regarding BMI and glycemic control in order to establish a clear preoperative recommendation for knee arthroplasty patients.

Another attractive strategy is to calculate a preoperative risk of PJI using a scoring system. The Mayo Clinic recently presented a prosthetic joint infection risk score, which demonstrated a good capacity to discriminate subjects who will develop a PJI from those who will not (C-index of 0.722$)^{5}$. It includes the BMI, presence of prior operations on the index joint, prior arthroplasty, immunosuppression, ASA score, and procedure duration ${ }^{5}$. After adding postoperative wound drainage to the previous variables, the 1-month post surgery risk score presents a C- index of 0.716 .

A recent study conducted in Korea described a prevalence of surgical site infections (SSI) in 161 of 6,848 cases $(2.35 \%)^{6}$. Interestingly, the authors suggested that the risk factors for SSI differ between total hip arthroplasty (THA), and TKA. Independent risk factors for SSI in TKA were male gender, and an operating room without laminar flow. More than 10 procedures per month was a protective factor for knee $\mathrm{PJI}^{6}$.

A recent systematic review and meta-analysis indicate that, compared with osteoarthritis (OA) patients, patients with rheumatoid arthritis have a higher risk of infection following TKA ${ }^{7}$.

Another important risk factor is revision surgical procedures ${ }^{8)}$. A study from our institution revealed that the risk of infection among patients undergoing revisions was 10 -fold higher $(9 \%)^{8)}$ than for patients undergoing primary TKA $(0.5 \%-1 \%)^{9)}$. The risk was predicted by factors such as revision due to infection, higher Charlson comorbidity index, and diagnosis other than OA at the time of the primary procedure.

Understanding the risk factors for PJI, allows implementation of strategies that aims to reverse some of these potential risk factors and reduce the burden of infection.

\section{Preoperative Antibiotics}

One of the most effective strategies for the prevention of infection in modern orthopaedic literature is the administration of preoperative antibiotics ${ }^{10,11}$. In order to achieve optimal results, adequate concentration of antibiotics should be present during the entire time the incision is open, when the greatest risk for contamination is present ${ }^{10)}$. For predictable and rapid delivery of antibiotics, systematic intravenous (IV) administration is the method of choice ${ }^{12)}$.

Conflicting opinions exist as to what the optimal time window for prophylaxis administration should be ${ }^{12}$. Some studies have shown that the best time for administration is within 30 minutes of incision ${ }^{13)}$, while others support administration within 30-59 minutes ${ }^{14}$. Based on The American Academy of Orthopaedic Surgeons (AAOS) and The Centers for Disease Control (CDC) guidelines, prophylactic antibiotics should be administered within one hour before the surgical incision ${ }^{15}$. When a proximal tourniquet is used, administration of the entire dose of antibiotic should occur before inflation of the tourniquet ${ }^{12,15)}$. Duration of antibiotic coverage should not exceed 24 hours postoperatively ${ }^{15}$, doing so may increase the risk for adverse effects of antibiotics without proven benefits ${ }^{15,16}$. Avoiding unnecessary antibiotic use will also minimize the risk ofbacterial resistance ${ }^{17}$. Furthermore, intraoperative dose of antibiotic should be repeated if there is significant blood loss, or if operative time exceeds two times the half-life of antibiotic ${ }^{15,18)}$.

Currently, the most widely used prophylactic antibiotics for prevention of PJI are first and second generation cephalosporins because of their excellent tissue penetration, bioavailability, and coverage against common organisms such as Staphylococcus species, and enteric pathogens ${ }^{12}$. In patients undergoing orthopaedic procedures, cefuroxamine and cefazolin are the preferred antibiotics $^{14)}$. These antibiotics may not be appropriate in certain cases, and the addition of vancomycin may be warranted ${ }^{12,15)}$. AAOS guidelines recommend vancomycin in facilities with methicillinresistant staphylococcus aureus (MRSA) outbreaks, and in patients with known MRSA colonization ${ }^{15}$. Additionally, vancomycin should be considered in institutionalized patients (dialysisdependent patients and nursing home residents), and health care workers ${ }^{12}$. Patients with a documented anaphylactic reaction to penicillin can either receive clindamycin or vancomycin ${ }^{12,15)}$, however, our institution prefers vancomycin to avoid the potential for clindamycin-associated clostridium difficile enteritis ${ }^{12)}$.

\section{Skin Preparation}

Preoperative skin preparation is of common practice in the orthopaedic community. Skin preparation prior to surgery includes skin decolonization, antisepsis, hand washing by the surgeon, and hair removal. Ample evidence exists in support of the role of preoperative cleansing in reduction of skin bacteria load ${ }^{19,20)}$, but how this translates to prevention of SSI is unclear.

Preoperative showering or cleansing with an antiseptic agent at 
least the night before a surgical procedure has been recommended by the $\mathrm{CDC}^{21}$. In two prospective consecutive series, patients who used chlorhexidine gluconate $(\mathrm{CHG})$ impregnated wipes the night before, and the morning of the surgery had a lower incidence of SSI as compared with those who did not comply to the protocol in both THA and TKA procedures. In contrast, a Cochrane review of 7 randomized trials concluded that preoperative showering with CHG did not reduce the rate of SSI as compared to a no shower group or placebo group ${ }^{22}$. Despite conflicting data regarding preoperative showers reducing SSI incidence, the simplicity and cost-effectiveness of this method justifies its current recommendation ${ }^{23)}$.

Regarding skin preoperative disinfection, the discussion has focused on the selection of an optimal antiseptic agent ${ }^{12)}$. The main types of antiseptic agents are: alcohol based solutions, povidoneiodine and $\mathrm{CHG}^{24,25)}$. In studies comparing $\mathrm{CHG}$ and povidoneiodine the results are conflicting. Darouiche et al. ${ }^{26)}$ demonstrated that $\mathrm{CHG}$ in alcohol was superior in reducing the rate of SSI as compared to aqueous povidone-iodine. However, the iodine preparation in the study did not use an alcohol solvent, hence allowing for the possibility that alcohol may play a role. In fact, in a study involving general surgery patients, povidone-iodine prepped patients had a lower rate of SSI when alcohol was used (either as a solvent or scrub) ${ }^{27)}$. Overall, the literature suggests some value in the combination of alcohol with antiseptic agents, and that CHG combined with alcohol may be superior to other combinations $^{26,28,29)}$.

In regards to preoperative hair removal, the CDC recommends that it should be done immediately before the procedure, and electric clippers are preferred over razor blades ${ }^{12,21)}$. Tanner et al. ${ }^{30)}$ conducted a meta-analysis that showed that electric clippers were associated with fewer SSIs than with razor shaving.

Hand washing by the surgeon and medical personnel is a difficult topic to evaluate due to the variability in the literature regarding duration, and optimal antiseptic agent. One study examining surgical scrub time, and subsequent bacterial growth found no significant difference between a 2 or a 3 minute scrub ${ }^{31}$. Currently, The Association of Perioperative Registered Nurses states that a 3-4 minute scrub is as effective as a 5 minute scrub, while the CDC recommends $2-5$ minutes ${ }^{21}$. Data on hand rub (alcohol based) and hand scrub agents suggest no significant difference in efficacy between the two ${ }^{32}$. A trial of 4,387 patients who underwent clean, and clean contaminated surgery using either traditional hand scrubbing techniques or waterless, alcohol-based antiseptics showed no difference in SSI rates ${ }^{33)}$. Based on current literature, medical personnel should consider a minimum dura- tion of 2-3 minutes for surgical hand antisepsis using either hand rub or hand scrub solution ${ }^{31-33)}$.

\section{Diagnosis of Periprosthetic Joint Infection}

The diagnosis of PJI should be suspected in all patients evaluated for a painful TKA. The AAOS established diagnostic guidelines that help identify PJI of the knee ${ }^{34)}$. The measurement of serum markers such as C-reactive protein (CRP), and the erythrocyte sedimentation rate (ESR) is highly valuable in reaching a diagnosis of PJI. It must be noted that these tests are not diagnostic independently. According to the $\mathrm{AAOS}^{34)}$ and the Infectious Diseases Society of America guidelines ${ }^{35)}$, if serum markers are elevated, a joint aspiration should be performed. Our institution always includes cytochemical fluid analysis, and two cultures after every knee aspiration. More recently, we have added the evaluation of the leukocyte esterase test also ${ }^{36}$.

We encourage the surgeons to be cognizant of the Musculoskeletal Infection Society (MSIS) PJI diagnostic criteria, that aim to provide a standard definition of $\mathrm{PJI}^{37)}$. The criteria can be used as a guide to establish a diagnosis when PJI is suspected. Taking into consideration the benefits, costs and risks of different strategies of PJI diagnosis, the use of serum markers followed by knee arthrocentesis is a highly efficient strategy.

A recent study from our group determined the thresholds for serum markers to diagnose PJI using the MSIS criteria. In early postoperative knee PJI, the ESR threshold was $54 \mathrm{~mm}$ /hour, and CRP was $23.5 \mathrm{mg} / \mathrm{L}$. In late-chronic knee PJI the thresholds were $46.5 \mathrm{~mm} /$ hour for ESR, and $23.5 \mathrm{mg} / \mathrm{L}$ for $\mathrm{CRP}^{38)}$. A study from another group established that the rate of false negatives was $9.2 \%$ for ESR, 5.3\% for CRP, and $11 \%$ for combined ESR and CRP when diagnosing knee PJI. The authors believe that one of the factors that may explain these observations is that some patients may not mount a sufficient immune response, especially in early postoperative infections ${ }^{39}$. For this reason, a joint aspiration should be conducted even if serum markers are normal in those cases with high clinical suspicion, or known risk factors for knee PJI.

Our group also presented the value of studying the CRP in the synovial fluid, which may be an additional tool to improve our diagnostic alternatives ${ }^{40)}$.

\section{Intraoperative Assessment in Every Revision Case}

From our standpoint and according to the recommendations made by the AAOS, surgeons should always perform a PJI diagnostic workup in every revision case. This process should start when the decision to undergo a revision is made, and the strate- 
gies described above are used. Once the patient is in the operation room, three main strategies can be employed: 1) cultures, 2) frozen sections, and 3) implant-related studies.

Concerning intraoperative cultures, one of the most important steps is to not withhold the preoperative antibiotics in revision cases. A multicenter randomized study, demonstrated that intraoperative cultures yielded the same organisms as preoperative cultures in 28 of 34 patients (82\%) randomized to receive antibiotics before the skin incision compared to 25 of 31 patients (81\%) randomized to receive antibiotics after obtaining operative cultures ${ }^{41}$. In regards to the type of cultures that should be obtained during the surgery, a study conducted in our institution demonstrated that tissue cultures are better than swab cultures. Tissue cultures demonstrated higher sensitivity, specificity, positive predictive value (PPV), and negative predictive value (NPV) for diagnosing PJI than swab cultures. Swab cultures had more false-negative and false-positive results than tissue cultures ${ }^{42)} \mathrm{An}$ other important strategy is to obtain the synovial fluid culture in blood culture flasks, due to its higher sensitivity, specificity, and both PPV and NPV for diagnosis of PJI when compared with standard tissue and swab samples ${ }^{43)}$.

Regarding the use of frozen sections, it must be noted that they represent one of the diagnostic criteria in the MSIS definition. A recent systematic review and meta-analysis by Tsaras et al. ${ }^{44)}$ showed that intraoperative frozen sections are helpful in the diagnosis of culture-positive PJI, but had moderate accuracy in ruling out this diagnosis. According to the authors, frozen section is especially valuable if the suspicion of infection remains high even after a negative preoperative evaluation. The optimum diagnostic threshold (number of polymorphonuclear leukocyte per highpower field) could not be discerned in this study.

Implant sonication is proving to be an interesting technology ${ }^{45}$. Its role has not been completely determined in the diagnostic work-up of PJI, especially considering the costs associated.

\section{How to Improve the Utility of Cultures?}

Cultures of tissue and synovial fluid obtained from an affected joint play a major role in both the diagnosis and treatment of $\mathrm{PJI}^{12}$. These cultures are used to confirm, and not to screen for $\mathrm{PJI}^{12)}$. Currently, diagnosis of PJI can be made from two separate fluid or tissue samples from an affected joint ${ }^{37}$. Not only do cultures confirm a PJI diagnosis, they also allow for sensitivity-guided treatment. However, in $7 \%$ to $12 \%$ of PJI cases cultures may be negative even when clear signs of infection are present ${ }^{46,47}$.

Negative cultures may be caused by a variety of reasons, including inappropriate collection of sample, use of antimicrobial therapy prior to collection, short incubation duration, and possible fungal or mycobacterial infection ${ }^{12)}$. In the setting of a true PJI, negative cultures limit the ability to tailor antibiotic treatment and may hinder justification for revision surgery ${ }^{12)}$. Thus, attention should be directed at decreasing the incidence of negative cultures.

Strategy for obtaining positive cultures includes withholding aspiration of joints for at least two weeks prior to sample collection, followed by a prolonged period of incubation ${ }^{12,46}$. A study by Schaefer et al ${ }^{48)}$ described that prolonged incubation for at least 14 days increased the identification of organisms that would otherwise remain culture negative. Certain pathogens that are difficult to isolate using traditional cultures include fungi, mycobacteria, and organisms encapsulated in biofilm ${ }^{46)}$. Proper media should be used in cases where fungi or mycobacteria are suspected $^{12)}$. A repeat aspiration should be considered in the event of inconclusive preoperative aspiration, negative cultures, elevated inflammatory markers, and high PJI suspicion ${ }^{12}$.

\section{Irrigation and Debridement: When and How?}

The use of irrigation and debridement (I\&D) with or without modular exchange as an appropriate alternative for treatment of PJI remains controversial. This less invasive procedure is commonly used despite its rate of success ranging between $0 \%$ and $89 \%{ }^{49)}$. I\&D provides the option for a less complex surgery, and lower cost when compared to two-staged procedures ${ }^{50)}$. The potential advantages of I\&D over two-stage exchange justify its continued practice and further investigation of factors that predict its optimal use.

I\&D has been considered a viable option for early postoperative or late hematogenous infections ${ }^{511}$. Previous studies have suggested that the ability of I\&D to control infection may be related to its timing relative to index total joint arthroplasty, and duration of symptoms ${ }^{52-54)}$. Hartman et al. ${ }^{52)}$ found significant improvement in I\&D success rates if used within 4 weeks of index knee surgery. In addition to short duration of symptoms, the literature suggests a higher I\&D success rate in healthier patients, and in infections with low virulent organisms ${ }^{55-58)}$. Azzam et al. ${ }^{59)}$ described that patients with a higher ASA score, had higher failure rates. Furthermore, several studies recognize Staphylococcal infections as a risk factor for failure in $I \& D^{60)}$. Thus, I\&D should be considered in immunologically optimized patients with acute onset of symptoms infected with low virulent organisms.

Once the decision to perform an I\&D procedure is made, preoperative optimization of the patient should be attempted before proceeding ${ }^{12}$. In the operating room, aggressive debridement 
of all foreign and affected periarticular tissue should be performed ${ }^{12)}$. At our institution, retained components are scrubbed with Dakin's solution and using a new, clean instrument for each collection, at least 3 tissue samples are then obtained ${ }^{12}$. Thorough irrigation using low pressure pulse lavage or bulb irrigation of the joint with up to 9 liters of solution is performed ${ }^{12,61,62)}$. Finally, gloves, gowns and surgical setup should be changed between the I\&D procedure, and the modular component exchange ${ }^{12)}$.

\section{Revision in One Stage: When and How?}

One-stage exchange arthroplasty for PJI has become the subject of interest due to potential advantages over two-stage exchange. Main advantages that proponents focus on are the need for a single operation, decreased morbidity, lower cost, and improved functional results ${ }^{63-65)}$. Regarding efficacy of the procedure, several retrospective studies have reported rates of infection control between $73 \%$ and $93 \%{ }^{66-69)}$.

Although advantages may exist in one-stage exchange, studies emphasize that success of the procedure depends on both patient, and infection related factors ${ }^{12)}$. Jackson and Schmalzried ${ }^{70)}$ performed a literature review to determine when single-stage exchange is most successful in the setting of an infected hip. Variables associated with successful outcomes included the absence of wound complications after index THA, healthy patients, presence of methicillin-sensitive organisms, and organism susceptibility to antibiotic-laden bone cement ${ }^{70}$. Factors that predicted failure included polymicrobial infection, presence of gram negative organisms, and methicillin-resistant organisms ${ }^{70)}$. Furthermore, the following may be considered contraindications: systemic infection, severe soft tissue involvement, inability to identify a microorganism preoperatively, and presence of a sinus tract ${ }^{10,12,35,70-72)}$. Identifying ways to optimize results of one-stage exchange helps determine appropriate indications. At our institution, indications for one-stage exchange include: a healthy host, acute postoperative infection, susceptible organism, and adequate soft tissue coverage $^{12)}$.

In one-stage exchange arthroplasty, a patient undergoes a radical synovectomy, debridement of infected tissue and removal of foreign material (including prosthesis and cement) ${ }^{65}$. Multiple cultures should be taken for final culture analysis ${ }^{12}$. Prior to the reimplantation of the prosthesis, the patient should be reprepped, and a change of gloves and surgical instruments should occur $^{65}$. After implantation of the antibiotic impregnated cement, the wound is irrigated with dilute betadine solution before final closure ${ }^{65}$. Systemic antibiotics are usually given for a total of 6 weeks, beginning with 2 weeks of IV antibiotics followed by 4 weeks of oral antibiotics ${ }^{65)}$.

\section{Revision in Two Stages: When and How?}

The two-stage exchange arthroplasty is currently the most accepted procedure for the treatment of PJI in North America ${ }^{73-75)}$. Two-stage exchange involves resection of the implants, meticulous debridement and irrigation, placement of a temporary antibiotic-impregnated cement spacer, and delayed component reimplantation ${ }^{12,65}$. Although the main role of two-stage revision has been in chronic PJI management, it is increasingly considered in cases of acute PJI where initial I\&D or one-stage exchange procedures have failed ${ }^{12,76)}$. Literature on two-stage exchange reports variable success rates, and sufficient data directly comparing it to one-stage revision is lacking. However, a recent systematic review demonstrated an average success rate of $90 \%$ after two-stage exchange for knee prosthesis infection ${ }^{75)}$. The study also reported that two-stage exchange provided better outcomes than one-stage revision for septic knee prosthesis ${ }^{75}$.

Currently, there is insufficient data to provide clear indications for two-stage exchange ${ }^{35,70,72)}$. Infections with resistant organisms have been associated with higher failure rates in the treatment of $\mathrm{PJI}^{77,78)}$. Some studies suggest that two-stage exchange may be the preferred treatment for highly virulent organisms ${ }^{79,80)}$. Parvizi et al. ${ }^{79)}$ examined surgical treatment success of knee and hip MRSA infections, and reported infection control by I\&D and two-stage exchange as $37 \%$ and $75 \%$ respectively, suggesting superior outcomes with the latter. Furthermore, Oussedik et al. ${ }^{64)}$ reported significant bone loss, and soft-tissue compromise as factors in favor of a two-stage exchange over a one-stage revision. Insufficient soft-tissue coverage may be an indication for two-stage exchange, especially if time is required for flap development. Currently at our institution, common indications for this procedure are as follows: chronic PJI, failed I\&D, and acute infections associated with an immunocompromised host or virulent organism ${ }^{12)}$.

Two-stage exchange begins with thorough removal of infected tissue and foreign material, followed by irrigation ${ }^{12}$. The first stage involves insertion of either a static or dynamic antibioticimpregnated spacer ${ }^{12}$, most commonly using vancomycin, tobramycin, and gentamicin as the antibiotic ${ }^{12,81)}$. Postoperatively, the patient receives a course of antibiotic treatment, usually for 6 weeks $^{82}$, followed by reimplantation of a new prosthesis when the clinician deems the infection resolved ${ }^{12,83-85}$. A combination of clinical judgment, aspiration, and serological data can aid the clinician's decision on appropriate time for reimplantation ${ }^{12,82}$. 


\section{Patient Information about Their Prognosis}

A patient diagnosed with a knee PJI should be informed about the prognosis. Providing information is key in maintaining realistic expectations, and avoiding medicolegal issues. Ideally, the patient's family should also be involved during the presentation of prognostic information.

Although the term "successful treatment" in PJI has been widely used, until recently, its definition was non-uniform. Our group found more than 10 different definitions of success in the current literature ${ }^{86)}$, thus, we decided to create a Delphi-based consensus definition. The study was published recently ${ }^{86)}$, and it described success as 1) infection eradication (characterized by a healed wound without fistula, drainage, or pain), and no infection recurrence caused by the same organism strain, 2) no subsequent surgical intervention for infection after reimplantation surgery; and, 3) no occurrence of PJI-related mortality (by causes such as sepsis, necrotizing fasciitis). We expect that in the near future this definition will help us better present prognostic information, and the overall probability to succeed to optimize patient understanding.

In terms of infection eradication, according to a meta-analysis by Jamsen et al. ${ }^{87)}$, the failure to eradicate infection after treatment of a periprosthetic knee infection ranged from $0 \%-31 \%$. Recurrent infections occurred in $0 \%-18 \%$, and new infections varied from $0 \%-31 \%{ }^{87)}$. Regarding functional outcomes, Barrack et $\mathrm{al}^{88)}$, in a multicenter study of surgical outcomes following revision knee arthroplasty, patients demonstrated a lower Knee Society Score (KSS) in their cohort of septic revisions compared with revisions for aseptic failure. Concerning the radical management of failed TKA infection treatment, Chen et al. ${ }^{89)}$ demonstrated that patients with knee fusions had better functional scores than knee amputations.

In TKA revisions, Barrack et al. ${ }^{88)}$ demonstrated that revisions due to infection had no differences regarding patients' satisfaction compared with aseptic revision cases. This fact has been supported in a subsequent study by Patil et al..$^{90)}$.

\section{Conclusions}

Knee PJI is a devastating complication that is faced by every knee arthroplasty surgeon. We suggest that PJI should be combated in an organized way, establishing institutional or even national protocols in order to decrease the variability in its prevention and management. Based on current knowledge, we highly recommend the inclusion of these ten strategies as preoperative, intraoperative, and postoperative measures to minimize the risk and consequences of periprosthetic knee infection.

\section{Conflict of Interest}

Javad Parvizi: Speakers bureau/paid presentations: Elsevier, Wolters Kluwer, Slack, Datarace; Consulting: Zimmer, Smith\& Nephew, Convatech, TissueGene, Ceramtec, Emovi, 3M, Cadence, Medtronic, Pfizer; Stock: SmarTech, Hip Innovation Technology; Medical/Orthoapedics publications editorials/governing board: Journal of Athroplasty, Journal of Bone and Joint Surgery, Bone and Joint Journal; Service on Committees/Boards: Philadelphia Orthopaedics, Eastern Orthopaedics, CD Diagnostics, United Healthcare, Magnifi Group (Publishers), 3M.

\section{References}

1. Namba RS, Inacio MC, Paxton EW. Risk factors associated with deep surgical site infections after primary total knee arthroplasty: an analysis of 56,216 knees. J Bone Joint Surg Am. 2013;95:775-82.

2. Workgroup of the American Association of Hip and Knee Surgeons Evidence Based Committee. Obesity and total joint arthroplasty: a literature based review. J Arthroplasty. 2013;28:714-21.

3. Jamsen E, Nevalainen P, Eskelinen A, Huotari K, Kalliovalkama J, Moilanen T. Obesity, diabetes, and preoperative hyperglycemia as predictors of periprosthetic joint infection: a single-center analysis of 7181 primary hip and knee replacements for osteoarthritis. J Bone Joint Surg Am. 2012;94:e101.

4. Iorio R, Williams KM, Marcantonio AJ, Specht LM, Tilzey JF, Healy WL. Diabetes mellitus, hemoglobin A1C, and the incidence of total joint arthroplasty infection. J Arthroplasty. 2012;27:726-9.

5. Berbari EF, Osmon DR, Lahr B, Eckel-Passow JE, Tsaras G, Hanssen AD, Mabry T, Steckelberg J, Thompson R. The Mayo prosthetic joint infection risk score: implication for surgical site infection reporting and risk stratification. Infect Control Hosp Epidemiol. 2012;33:774-81.

6. Song KH, Kim ES, Kim YK, Jin HY, Jeong SY, Kwak YG, Cho YK, Sung J, Lee YS, Oh HB, Kim TK, Koo KH, Kim EC, Kim JM, Choi TY, Kim HY, Choi HJ, Kim HB. Differences in the risk factors for surgical site infection between total hip arthroplasty and total knee arthroplasty in the Korean Nosocomial Infections Surveillance System (KONIS). Infect Control Hosp Epidemiol. 2012;33:1086-93. 
7. Ravi B, Escott B, Shah PS, Jenkinson R, Chahal J, Bogoch E, Kreder H, Hawker G. A systematic review and meta-analysis comparing complications following total joint arthroplasty for rheumatoid arthritis versus for osteoarthritis. Arthritis Rheum. 2012;64:3839-49.

8. Mortazavi SM, Schwartzenberger J, Austin MS, Purtill JJ, Parvizi J. Revision total knee arthroplasty infection: incidence and predictors. Clin Orthop Relat Res. 2010;468:20529.

9. Pulido L, Ghanem E, Joshi A, Purtill JJ, Parvizi J. Periprosthetic joint infection: the incidence, timing, and predisposing factors. Clin Orthop Relat Res. 2008;466:1710-5.

10. Bratzler DW, Houck PM; Surgical Infection Prevention Guideline Writers Workgroup. Antimicrobial prophylaxis for surgery: an advisory statement from the National Surgical Infection Prevention Project. Am J Surg. 2005;189:395404.

11. Prokuski L. Prophylactic antibiotics in orthopaedic surgery. J Am Acad Orthop Surg. 2008;16:283-93.

12. Parvizi J, Kerr GJ, Glynn A, Higuera CA, Hansen EN. Periprosthetic joint infection: practical management guide. 1st ed. New Delhi: Jaypee Brothers Medical Publishers; 2013. p121.

13. van Kasteren ME, Mannien J, Ott A, Kullberg BJ, de Boer AS, Gyssens IC. Antibiotic prophylaxis and the risk of surgical site infections following total hip arthroplasty: timely administration is the most important factor. Clin Infect Dis. 2007;44:921-7.

14. Weber WP, Marti WR, Zwahlen M, Misteli H, Rosenthal R, Reck S, Fueglistaler P, Bolli M, Trampuz A, Oertli D, Widmer AF. The timing of surgical antimicrobial prophylaxis. Ann Surg. 2008;247:918-26.

15. American Academy of Orthopaedic Surgeons. Recommendations for the Use of Intravenous Antibiotic Prophylaxis Information Statement [Internet]. IL: American Academy of Orthopaedic Surgeons; c2013 [cited 2013 Aug 10]. Available from: http://www.aaos.org/about/papers/advistmt/1027.asp.

16. McDonald M, Grabsch E, Marshall C, Forbes A. Singleversus multiple-dose antimicrobial prophylaxis for major surgery: a systematic review. Aust N Z J Surg. 1998;68:38896.

17. Harbarth S, Samore MH, Lichtenberg D, Carmeli Y. Prolonged antibiotic prophylaxis after cardiovascular surgery and its effect on surgical site infections and antimicrobial resistance. Circulation. 2000;101:2916-21.

18. Page CP, Bohnen JM, Fletcher JR, McManus AT, Solomkin
JS, Wittmann DH. Antimicrobial prophylaxis for surgical wounds. Guidelines for clinical care. Arch Surg. 1993;128:7988.

19. Kalmeijer MD, Coertjens H, van Nieuwland-Bollen PM, Bogaers-Hofman D, de Baere GA, Stuurman A, van Belkum A, Kluytmans JA. Surgical site infections in orthopedic surgery: the effect of mupirocin nasal ointment in a doubleblind, randomized, placebo-controlled study. Clin Infect Dis. 2002;35:353-8.

20. Perl TM, Cullen JJ, Wenzel RP, Zimmerman MB, Pfaller MA, Sheppard D, Twombley J, French PP, Herwaldt LA; Mupirocin And The Risk Of Staphylococcus Aureus Study Team. Intranasal mupirocin to prevent postoperative Staphylococcus aureus infections. N Engl J Med. 2002;346:1871-7.

21. Mangram AJ, Horan TC, Pearson ML, Silver LC, Jarvis WR. Guideline for Prevention of Surgical Site Infection, 1999. Centers for Disease Control and Prevention (CDC) Hospital Infection Control Practices Advisory Committee. Am J Infect Control. 1999;27:97-132.

22. Webster J, Osborne S. Preoperative bathing or showering with skin antiseptics to prevent surgical site infection. Cochrane Database Syst Rev. 2007;(2):CD004985.

23. Zywiel MG, Daley JA, Delanois RE, Naziri Q, Johnson AJ, Mont MA. Advance pre-operative chlorhexidine reduces the incidence of surgical site infections in knee arthroplasty. Int Orthop. 2011;35:1001-6.

24. O'Grady NP, Alexander M, Dellinger EP, Gerberding JL, Heard SO, Maki DG, Masur H, McCormick RD, Mermel LA, Pearson ML, Raad II, Randolph A, Weinstein RA. Guidelines for the prevention of intravascular catheterrelated infections: the Hospital Infection Control Practices Advisory Committee, Center for Disease Control and Prevention, U.S. Pediatrics. 2002;110:e51.

25. Olson LK, Morse DJ, Duley C, Savell BK. Prospective, randomized in vivo comparison of a dual-active waterless antiseptic versus two alcohol-only waterless antiseptics for surgical hand antisepsis. Am J Infect Control. 2012;40:155-9.

26. Darouiche RO, Wall MJ Jr, Itani KM, Otterson MF, Webb AL, Carrick MM, Miller HJ, Awad SS, Crosby CT, Mosier MC, Alsharif A, Berger DH. Chlorhexidine-Alcohol versus Povidone-Iodine for Surgical-Site Antisepsis. N Engl J Med. 2010;362:18-26.

27. Swenson BR, Hedrick TL, Metzger R, Bonatti H, Pruett TL, Sawyer RG. Effects of preoperative skin preparation on postoperative wound infection rates: a prospective study of 3 skin preparation protocols. Infect Control Hosp Epidemiol. 
2009;30:964-71.

28. Keblish DJ, Zurakowski D, Wilson MG, Chiodo CP. Preoperative skin preparation of the foot and ankle: bristles and alcohol are better. J Bone Joint Surg Am. 2005;87:986-92.

29. Ostrander RV, Botte MJ, Brage ME. Efficacy of surgical preparation solutions in foot and ankle surgery. J Bone Joint Surg Am. 2005;87:980-5.

30. Tanner J, Norrie P, Melen K. Preoperative hair removal to reduce surgical site infection. Cochrane Database Syst Rev. 2011;(11):CD004122.

31. Wheelock SM, Lookinland S. Effect of surgical hand scrub time on subsequent bacterial growth. AORN J. 1997;65:1087-92.

32. Tanner J, Swarbrook S, Stuart J. Surgical hand antisepsis to reduce surgical site infection. Cochrane Database Syst Rev. 2008;(1):CD004288.

33. Parienti JJ, Thibon P, Heller R, Le Roux Y, von Theobald P, Bensadoun H, Bouvet A, Lemarchand F, Le Coutour X; Antisepsie Chirurgicale des mains Study Group. Hand-rubbing with an aqueous alcoholic solution vs traditional surgical hand-scrubbing and 30-day surgical site infection rates: a randomized equivalence study. JAMA. 2002;288:722-7.

34. Adopted by the American Academy of Orthopaedic Surgeons Board of Directors. The diagnosis of periprosthetic joint infections of the hip and knee: guideline and evidence report [Internet]. IL: American Academy of Orthopaedic Surgeons; c2013 [cited 2012 Feb 2]. Available from: http:// www.aaos.org/research/guidelines/PJIguideline.pdf.

35. Osmon DR, Berbari EF, Berendt AR, Lew D, Zimmerli W, Steckelberg JM, Rao N, Hanssen A, Wilson WR; Infectious Diseases Society of America. Executive summary: diagnosis and management of prosthetic joint infection: clinical practice guidelines by the Infectious Diseases Society of America. Clin Infect Dis. 2013;56:1-10.

36. Aggarwal VK, Tischler E, Ghanem E, Parvizi J. Leukocyte esterase from synovial fluid aspirate: a technical note. J Arthroplasty. 2013;28:193-5.

37. Parvizi J, Zmistowski B, Berbari EF, Bauer TW, Springer BD, Della Valle CJ, Garvin KL, Mont MA, Wongworawat MD, Zalavras CG. New definition for periprosthetic joint infection: from the Workgroup of the Musculoskeletal Infection Society. Clin Orthop Relat Res. 2011;469:2992-4.

38. Alijanipour P, Bakhshi H, Parvizi J. Diagnosis of periprosthetic joint infection: the threshold for serological markers. Clin Orthop Relat Res. 2013;471:3186-95.

39. Johnson AJ, Zywiel MG, Stroh A, Marker DR, Mont MA.
Serological markers can lead to false negative diagnoses of periprosthetic infections following total knee arthroplasty. Int Orthop. 2011;35:1621-6.

40. Parvizi J, McKenzie JC, Cashman JP. Diagnosis of periprosthetic joint infection using synovial C-reactive protein. J Arthroplasty. 2012;27(8 Suppl):12-6.

41. Tetreault MW, Wetters NG, Aggarwal V, Mont M, Parvizi J, Della Valle CJ. The Chitranjan Ranawat Award: should prophylactic antibiotics be withheld before revision surgery to obtain appropriate cultures? Clin Orthop Relat Res. 2013 Apr 30 [Epub]. http://dx.doi.org/ 10.1007/s11999-013-30165.

42. Aggarwal VK, Higuera C, Deirmengian G, Parvizi J, Austin MS. Swab cultures are not as effective as tissue cultures for diagnosis of periprosthetic joint infection. Clin Orthop Relat Res. 2013;471:3196-203.

43. Font-Vizcarra L, Garcia S, Martínez-Pastor JC, Sierra JM, Soriano A. Blood culture flasks for culturing synovial fluid in prosthetic joint infections. Clin Orthop Relat Res. 2010;468:2238-43.

44. Tsaras G, Maduka-Ezeh A, Inwards CY, Mabry T, Erwin PJ, Murad MH, Montori VM, West CP, Osmon DR, Berbari EF. Utility of intraoperative frozen section histopathology in the diagnosis of periprosthetic joint infection: a systematic review and meta-analysis. J Bone Joint Surg Am. 2012;94:170011.

45. Gomez E, Cazanave C, Cunningham SA, GreenwoodQuaintance KE, Steckelberg JM, Uhl JR, Hanssen AD, Karau MJ, Schmidt SM, Osmon DR, Berbari EF, Mandrekar J, Patel R. Prosthetic joint infection diagnosis using broad-range PCR of biofilms dislodged from knee and hip arthroplasty surfaces using sonication. J Clin Microbiol. 2012;50:3501-8.

46. Higuera C, Parvizi J. Culture negative PJI periprosthetic joint infection: strategies to improve yield [Internet]. Thorofare, NJ: Healio; 2012 [cited 2013 Aug 10]. Available from: http:// www.healio.com/orthopedics/hip/news/print/orthopedicstoday/\%7B92241423-c80c-4320-bb54-76a3f8e96978\%7D/ culture-negative-pji-periprosthetic-joint-infection-strategies-toimprove-yield.

47. Parvizi J, Ghanem E, Menashe S, Barrack RL, Bauer TW. Periprosthetic infection: what are the diagnostic challenges? J Bone Joint Surg Am. 2006;88 Suppl 4:138-47.

48. Schafer P, Fink B, Sandow D, Margull A, Berger I, Frommelt L. Prolonged bacterial culture to identify late periprosthetic joint infection: a promising strategy. Clin Infect Dis. 2008;47:1403-9. 
49. Romano CL, Manzi G, Logoluso N, Romano D. Value of debridement and irrigation for the treatment of peri-prosthetic infections: a systematic review. Hip Int. 2012;22 Suppl 8:S1924.

50. Sukeik M, Patel S, Haddad FS. Aggressive early debridement for treatment of acutely infected cemented total hip arthroplasty. Clin Orthop Relat Res. 2012;470:3164-70.

51. Odum SM, Fehring TK, Lombardi AV, Zmistowski BM, Brown NM, Luna JT, Fehring KA, Hansen EN; Periprosthetic Infection Consortium. Irrigation and debridement for periprosthetic infections: does the organism matter? J Arthroplasty. 2011;26(6 Suppl):114-8.

52. Hartman MB, Fehring TK, Jordan L, Norton HJ. Periprosthetic knee sepsis. The role of irrigation and debridement. Clin Orthop Relat Res. 1991;(273):113-8.

53. Marculescu CE, Berbari EF, Hanssen AD, Steckelberg JM, Harmsen SW, Mandrekar JN, Osmon DR. Outcome of prosthetic joint infections treated with debridement and retention of components. Clin Infect Dis. 2006;42:471-8.

54. Silva M, Tharani R, Schmalzried TP. Results of direct exchange or debridement of the infected total knee arthroplasty. Clin Orthop Relat Res. 2002;(404):125-31.

55. Koyonos L, Zmistowski B, Della Valle CJ, Parvizi J. Infection control rate of irrigation and débridement for periprosthetic joint infection. Clin Orthop Relat Res. 2011;469:3043-8.

56. Lora-Tamayo J, Murillo O, Iribarren JA, Soriano A, SanchezSomolinos M, Baraia-Etxaburu JM, Rico A, Palomino J, Rodriguez-Pardo D, Horcajada JP, Benito N, Bahamonde A, Granados A, del Toro MD, Cobo J, Riera M, Ramos A, Jover-Saenz A, Ariza J; REIPI Group for the Study of Prosthetic Infection. A large multicenter study of methicillinsusceptible and methicillin-resistant Staphylococcus aureus prosthetic joint infections managed with implant retention. Clin Infect Dis. 2013;56:182-94.

57. Tsukayama DT, Estrada R, Gustilo RB. Infection after total hip arthroplasty: a study of the treatment of one hundred and six infections. J Bone Joint Surg Am. 1996;78:512-23.

58. Zimmerli W, Widmer AF, Blatter M, Frei R, Ochsner PE. Role of rifampin for treatment of orthopedic implantrelated staphylococcal infections: a randomized controlled trial: Foreign-Body Infection (FBI) Study Group. JAMA. 1998;279:1537-41.

59. Azzam KA, Seeley M, Ghanem E, Austin MS, Purtill JJ, Parvizi J. Irrigation and debridement in the management of prosthetic joint infection: traditional indications revisited. J Arthroplasty. 2010;25:1022-7.
60. Deirmengian C, Greenbaum J, Lotke PA, Booth RE Jr, Lonner JH. Limited success with open debridement and retention of components in the treatment of acute Staphylococcus aureus infections after total knee arthroplasty. J Arthroplasty. 2003;18(7 Suppl 1):22-6.

61. Munoz-Mahamud E, Garcia S, Bori G, Martinez-Pastor JC, Zumbado JA, Riba J, Mensa J, Soriano A. Comparison of a low-pressure and a high-pressure pulsatile lavage during debridement for orthopaedic implant infection. Arch Orthop Trauma Surg. 2011;131:1233-8.

62. Schwechter EM, Folk D, Varshney AK, Fries BC, Kim SJ, Hirsh DM. Optimal irrigation and debridement of infected joint implants: an in vitro methicillin-resistant Staphylococcus aureus biofilm model. J Arthroplasty. 2011;26(6 Suppl):109-13.

63. Buechel FF, Femino FP, D'Alessio J. Primary exchange revision arthroplasty for infected total knee replacement: a longterm study. Am J Orthop (Belle Mead NJ). 2004;33:190-8.

64. Oussedik SI, Dodd MB, Haddad FS. Outcomes of revision total hip replacement for infection after grading according to a standard protocol. J Bone Joint Surg Br. 2010;92:1222-6.

65. Singer J, Merz A, Frommelt L, Fink B. High rate of infection control with one-stage revision of septic knee prostheses excluding MRSA and MRSE. Clin Orthop Relat Res. 2012;470:1461-71.

66. von Foerster G, Kluber D, Kabler U. Mid- to long-term results after treatment of 118 cases of periprosthetic infections after knee joint replacement using one-stage exchange surgery. Orthopade. 1991;20:244-52.

67. Goksan SB, Freeman MA. One-stage reimplantation for infected total knee arthroplasty. J Bone Joint Surg Br. 1992;74:78-82.

68. Lu H, Kou B, Lin J. One-stage reimplantation for the salvage of total knee arthroplasty complicated by infection. Zhonghua Wai Ke Za Zhi. 1997;35:456-8.

69. Sofer D, Regenbrecht B, Pfeil J. Early results of one-stage septic revision arthroplasties with antibiotic-laden cement. A clinical and statistical analysis. Orthopade. 2005;34:592602.

70. Jackson WO, Schmalzried TP. Limited role of direct exchange arthroplasty in the treatment of infected total hip replacements. Clin Orthop Relat Res. 2000;(381):101-5.

71. Klouche S, Leonard P, Zeller V, Lhotellier L, Graff W, Leclerc P, Mamoudy P, Sariali E. Infected total hip arthroplasty revision: one- or two-stage procedure? Orthop Traumatol Surg Res. 2012;98:144-50. 
72. Parvizi J, Adeli B, Zmistowski B, Restrepo C, Greenwald AS. Management of periprosthetic joint infection: the current knowledge: AAOS exhibit selection. J Bone Joint Surg Am. 2012;94:e104.

73. Freeman MG, Fehring TK, Odum SM, Fehring K, Griffin WL, Mason JB. Functional advantage of articulating versus static spacers in 2-stage revision for total knee arthroplasty infection. J Arthroplasty. 2007;22:1116-21.

74. Meek RM, Masri BA, Dunlop D, Garbuz DS, Greidanus NV, McGraw R, Duncan CP. Patient satisfaction and functional status after treatment of infection at the site of a total knee arthroplasty with use of the PROSTALAC articulating spacer. J Bone Joint Surg Am. 2003;85:1888-92.

75. Romano CL, Gala L, Logoluso N, Romano D, Drago L. Twostage revision of septic knee prosthesis with articulating knee spacers yields better infection eradication rate than onestage or two-stage revision with static spacers. Knee Surg Sports Traumatol Arthrosc. 2012;20:2445-53.

76. Parvizi J, Zmistowski B, Adeli B. Periprosthetic joint infection: treatment options. Orthopedics. 2010;33(9):659.

77. Kilgus DJ, Howe DJ, Strang A. Results of periprosthetic hip and knee infections caused by resistant bacteria. Clin Orthop Relat Res. 2002;(404):116-24.

78. Salgado CD, Dash S, Cantey JR, Marculescu CE. Higher risk of failure of methicillin-resistant Staphylococcus aureus prosthetic joint infections. Clin Orthop Relat Res. 2007;461:48-53.

79. Parvizi J, Azzam K, Ghanem E, Austin MS, Rothman RH. Periprosthetic infection due to resistant staphylococci: serious problems on the horizon. Clin Orthop Relat Res. 2009;467:1732-9.

80. Walls RJ, Roche SJ, O'Rourke A, McCabe JP. Surgical site infection with methicillin-resistant Staphylococcus aureus after primary total hip replacement. J Bone Joint Surg Br. 2008;90:292-8.

81. Hanssen AD, Spangehl MJ. Practical applications of anti- biotic-loaded bone cement for treatment of infected joint replacements. Clin Orthop Relat Res. 2004;(427):79-85.

82. Glassman AH, Lachiewicz PF, Tanzer M. Orthopaedic knowledge update: hip and knee reconstruction 4. Rosemont, IL: American Academy of Orthopaedic Surgeons; 2011. p202-7.

83. Goldman RT, Scuderi GR, Insall JN. 2-stage reimplantation for infected total knee replacement. Clin Orthop Relat Res. 1996;(331):118-24.

84. Haleem AA, Berry DJ, Hanssen AD. Mid-term to long-term followup of two-stage reimplantation for infected total knee arthroplasty. Clin Orthop Relat Res. 2004;(428):35-9.

85. Insall JN, Thompson FM, Brause BD. Two-stage reimplantation for the salvage of infected total knee arthroplasty. J Bone Joint Surg Am. 1983;65:1087-98.

86. Diaz-Ledezma C, Higuera CA, Parvizi J. Success after treatment of periprosthetic joint infection: a Delphi-based international multidisciplinary consensus. Clin Orthop Relat Res. 2013;471:2374-82.

87. Jamsen E, Stogiannidis I, Malmivaara A, Pajamaki J, Puolakka T, Konttinen YT. Outcome of prosthesis exchange for infected knee arthroplasty: the effect of treatment approach. Acta Orthop. 2009;80:67-77.

88. Barrack RL, Engh G, Rorabeck C, Sawhney J, Woolfrey M. Patient satisfaction and outcome after septic versus aseptic revision total knee arthroplasty. J Arthroplasty. 2000;15:9903.

89. Chen AF, Kinback NC, Heyl AE, McClain EJ, Klatt BA. Better function for fusions versus above-the-knee amputations for recurrent periprosthetic knee infection. Clin Orthop Relat Res. 2012;470:2737-45.

90. Patil N, Lee K, Huddleston JI, Harris AH, Goodman SB. Aseptic versus septic revision total knee arthroplasty: patient satisfaction, outcome and quality of life improvement. Knee. 2010;17:200-3. 